\title{
PANCREATITE AGUDA LEVE: AVALIAÇÃO PELA ULTRA-SONOGRAFIA. ESTUDO PROSPECTIVO*
}

\author{
Márcio Martins Machadoํㅜ, Ana Cláudia Ferreira Rosa², Nestor de Barros ${ }^{3}$, Luciana \\ Mendes de Oliveira Cerri ${ }^{4}$, Letícia Martins Azeredo $^{5}$, Giovanni Guido Cerri ${ }^{6}$
}

\begin{abstract}
Resumo Neste estudo foram avaliados, por meio da ultra-sonografia, 21 pacientes com pancreatite aguda leve. Foram analisadas a presença ou ausência de hipoecogenicidade do pâncreas e a presença ou ausência de líquido peripancreático. Em 19 pacientes $(90,5 \%)$ foi identificada hipoecogenicidade pancreática, e em três $(15,8 \%)$ destes pacientes foi identificada a presença de pequena quantidade de líquido na pequena cavidade dos epíploons. Em dois pacientes $(9,5 \%)$ não se identificou qualquer alteração pancreática. Com relação à possível etiologia da pancreatite aguda, em 15 pacientes $(71,5 \%)$ pôde-se demonstrar a presença de colecistopatia calculosa, em quatro pacientes $(19,0 \%)$ havia história de alcoolismo crônico e não foram identificados cálculos na vesícula biliar, e em dois pacientes $(9,5 \%)$ não foi identificada qualquer causa aparente. Os autores concluem que a ultra-sonografia pode identificar alteração na maioria dos pacientes com pancreatite aguda leve e permite, ainda, o acompanhamento daqueles com pequenas coleções líquidas peripancreáticas.

Unitermos: Pancreatite aguda. Complicações. Ultra-sonografia.
\end{abstract}

Abstract Mild acute pancreatitis: ultrasound evaluation. A prospective study.

We analyzed the ultrasonographic findings of 21 patients with mild acute pancreatitis. The presence or absence of pancreatic hypoechogenicity and peripancreatic fluid collection was assessed. Pancreatic hypoechogenicity was identified in 19 patients $(90.5 \%)$ whereas small sac fluid collection was identified in $3(15.8 \%)$ of these patients. No abnormality was seen in 2 patients $(9.5 \%)$. Regarding the etiology of acute pancreatitis, cholelithiasis was identified in 15 patients $(71.5 \%)$, alcohol abuse was identified in 4 patients $(19.0 \%)$, and in 2 patients $(9.5 \%)$ no probable etiology could be found. The authors conclude that ultrasonography may identify abnormalities in the majority of patients with mild acute pancreatitis and can be used to assess patients with peripancreatic fluid collections.

Key words: Acute pancreatitis. Complications. Ultrasonography.

* Trabalho realizado no Departamento de Radiologia do Hospital das Clínicas da Faculdade de Medicina da Universidade de São Paulo (HC-FMUSP) e no Departamento de Radiologia do Hospital Sírio Libanês, São Paulo, SP.

1. Médico Radiologista do Hospital das Clínicas da Faculdade de Medicina da Universidade Federal de Goiás (UFG), Doutor em Radiologia pela FMUSP, Médico Radiologista Consultor do Departamento de Doenças do Aparelho Digestivo do Hospital Araújo Jorge (Hospital do Câncer) da Associação de Combate ao Câncer em Goiás, Membro Titular do Colégio Brasileiro de Radiologia (CBR).

2. Médica Radiologista do Hospital das Clínicas da Faculdade de Medicina da UFG, Pós-graduanda do Departamento de Radiologia daFMUSP, ex-Assistente do Departamento de Radiologia do Hospital Sírio Libanês,Membro Titular do CBR.

3. Professor Doutor do Departamento de Radiologia da FMUSP.

4. Médica Radiologista, Assistente Doutora do Departamento de Radiologia do Hospital Sírio Libanês e do Instituto de Radiologia (InRad) do HC-FMUSP, Chefe do Setor de UItra-Sonografia da Divisão de Clínica Urológica do HC-FMUSP.

5. Médica Estagiária da Divisão de Ultra-Sonografia do Departamento de Radiologia do Hospital Sírio Libanês.

6. Professor Titular do Departamento de Radiologia da FMUSP, Chefe do InRad/HC-FMUSP e Diretor da Divisão de Diagnóstico por Imagem do Instituto do Coração (InCor) do HC-FMUSP, Chefe do Departamento de Radiologia do Hospital Sírio Libanês.

Endereço para correspondência: Dr. Márcio Martins Machado. Rua 1027, nำ230, Ed. Fabiana, apto. 304. Goiânia GO, 74823-120. E-mail: marciommachado@ibest.com.br

Recebido para publicação em 28/1/2002. Aceito, após revisão, em 4/3/2002.

\section{INTRODUÇÃO}

A pancreatite aguda é uma afecção que tem amplo espectro de apresentação, podendo, inclusive, ser fatal. Em muitos casos manifesta-se com caráter leve e autolimitado, com desaparecimento dos sintomas e resolução completa dos casos em poucos dias. Não obstante, deve ser tratada com cuidado, tendo em vista a intensa dor que provoca e as possíveis complicações que podem advir ${ }^{(1)}$.

Os métodos de imagem assumem importância, na medida em que podem fornecer subsídios morfológicos para os eventos bioquímicos envolvidos na gênese da pancreatite aguda, corroborando o diagnóstico. Desta forma, os imagenologistas devem estar afeitos aos aspectos de apresentação da pancreatite aguda.

Neste estudo, os autores avaliam a utilidade da ultra-sonografia em identificar a hipoecogenicidade da glândula pancreática e a presença de líquido peripancreático, associados a episódios de pancreatite agudaleve.

\section{MATERIAL E MÉTODOS}

Os pacientes estudados foram examinados, prospectivamente, no Departamento de Radiologia da Faculdade de Medicina da Universidade de São Paulo e no Hospital Sírio Libanês, São Paulo, SP, no período de janeiro de 1998 a outubro de 2001. Foram avaliados 21 pacientes, sendo nove do sexo masculino e 12 do sexo feminino, com idade variando de 19 a 58 anos.

Os exames foram realizados em aparelhos Logiq 500 ou 700 (GE, Milwaukee, Wisconsin, EUA), Toshiba SSH-140 (Tóquio, Japão) e Sonoline-Elegra (Siemens Medical Systems, Issaquah, Washington, EUA). Os transdutores utilizados foram convexos e apresentavam freqüência central de 3,5 MHz.

Todos os 21 pacientes tinham dor abdominal importante e elevação dos níveis 
séricos de amilase, caracterizando o diagnóstico de pancreatite aguda. O diagnóstico clínico de pancreatite aguda leve foi baseado na análise, em conjunto, dos dados dos exames clínicos, critérios de Ranson $^{(\mathbf{2})}$ e Apache $\mathrm{II}^{(\mathbf{3 , 4})}$. Todos os pacientes apresentavam escore menor que 3 , segundo os critérios de Ranson, e menor que 8, segundo os critérios do Apache II, caracterizando a forma leve da pancreatite aguda. Somente fizeram parte do estudo pacientes que, mesmo apresentando a forma leve de pancreatite aguda, foram internados por pelo menos quatro dias, por decisão da equipe médica que acompanhava os pacientes.

Dessa forma, os pacientes submeteramse a pelo menos três exames de ultra-sonografia, um na admissão, outro de dois a quatro dias após o início dos sintomas, e o último logo antes da alta hospitalar. Quando se identificou líquido peripancreático, utilizaram-se exames seriados de ultra-sonografia até a demonstração do desaparecimento da coleção líquida.

Com relação ao pâncreas, este estudo foi delineado para que se avaliasse exclusivamente o aspecto ecográfico do pâncreas e a presença ou ausência de coleções líquidas peripancreáticas associadas. Embora subjetivo, o critério de hipoecogenicidade do pâncreas foi considerado quando se identificou diminuição da ecogenicidade característica da glândula pancreática. Foi dada ênfase, também, à presença ou ausência de colecistopatia calculosa.
Procurou-se, também, estabelecer se havia a presença de consumo crônico de bebida alcoólica destilada, assim como de qualquer outro fator que pudesse relacionar-se com o desenvolvimento de pancreatite aguda, como o uso de certos medicamentos (relacionados com episódios de pancreatite aguda), trauma pancreático ou alterações bioquímicas (como hipercalcemia ou hiperlipidemia).

\section{RESULTADOS}

Em 19 pacientes $(90,5 \%)$ foi observada a presença de hipoecogenicidade do pâncreas. Destes 19 pacientes com hipoecogenicidade pancreática, em 14 casos $(73,7 \%)$ a hipoecogenicidade era difusa (Figura 1), em dois (10,5\%) era mais acentuada na cabeça do pâncreas (Figura 2), e em três $(15,8 \%)$ era mais acentuada no corpo/cauda. Em três destes 19 pacientes com hipoecogenicidade pancreática foi identificada a presença de pequena quantidade de líquido na pequena cavidade dos epíploons (15,8\%) (Figura 3).

Com relação à possível etiologia da pancreatite aguda, foi identificada a presença de colecistopatia calculosa em 15 pacientes $(71,5 \%)$ (Figura 4$)$, sendo que em outros quatro pacientes $(19,0 \%)$ havia história de alcoolismo crônico e não foram identificados cálculos na vesícula biliar. Em dois pacientes (9,5\%) não foi identificado qualquer fator, quer do ponto de vista de imagem, de história clínica (por exemplo, uso de medicamentos ou trauma) ou bioquímico, que pudesse estar relacionado com o desenvolvimento do episódio de pancreatite aguda.

\section{DISCUSSÃO}

O diagnóstico de pancreatite aguda comumente é estabelecido pelos achados de dor súbita no abdome superior, náuseas e vômitos, e aumento dos níveis séricos de amilase $^{(\mathbf{1})}$. A pancreatite aguda pode se manifestar como formas mais graves ou mais brandas. Nas formas graves, chamadas genericamente de "hemorrágicas", os fenômenos de hemorragia e necrose dominam o quadro patológico, apresentando maiores índices de complicação e mortalidade. Nas formas brandas, chamadas genericamente de "edematosas", a glândula pancreática e os tecidos retroperitoneais se encontram edemaciados, estando o pâncreas infiltrado por células inflamatórias, podendo haver diminutos focos "microscópicos" de necrose. Portanto, é na forma hemorrágica que se encontra sangramento importante no interior do pâncreas, estendendo-se também para o retroperitônio, estando associado a extensas áreas de necrose pancreática $^{(\mathbf{1 , 5}-7)}$.

Clinicamente, temos também formas de pancreatite aguda leves e graves. Evidentemente, predizer como evoluirão os episódios de pancreatite aguda é algo que vem estimulando investigadores de diversos serviços no decorrer dos anos ${ }^{(\mathbf{2 - 4 , 6 , 8 , 9 )} \text {. }}$

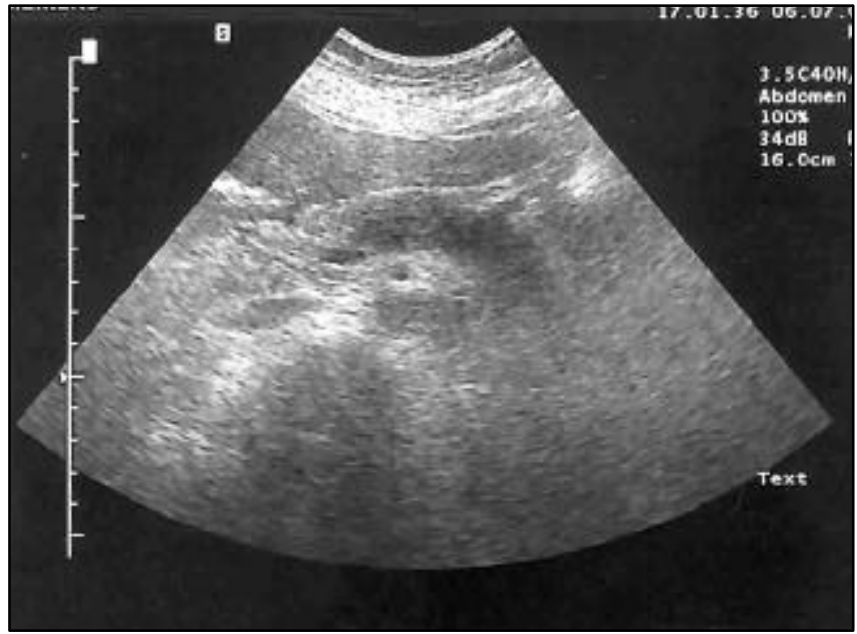

Figura 1. Pancreatite aguda difusa. Imagem ultra-sonográfica demonstrando aumento de volume e hipoecogenicidade do pâncreas difusamente.

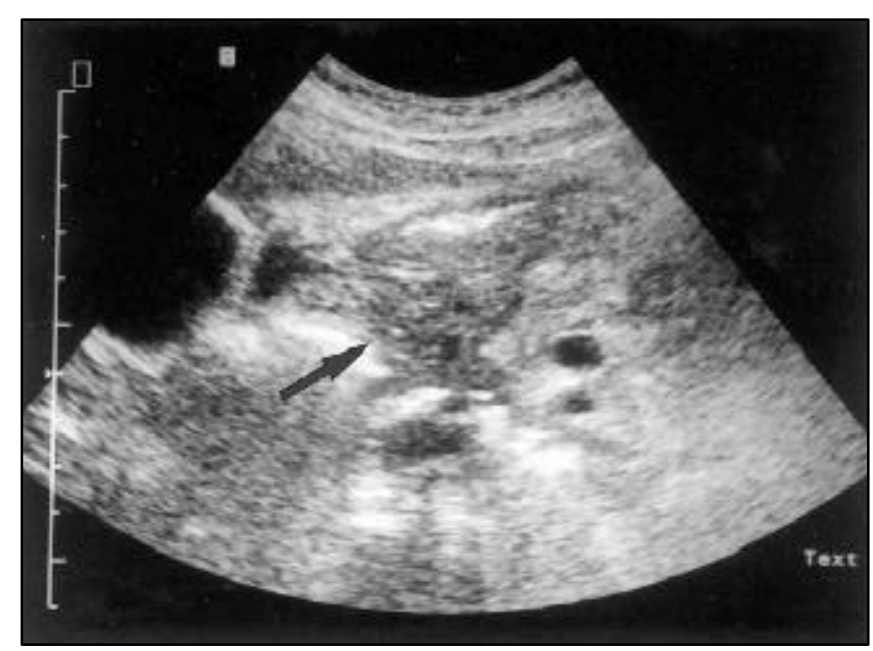

Figura 2. Imagem ultra-sonográfica transversal ao nível do pâncreas. A ultrasonografia evidencia aumento das dimensões na região cefálica, associado a diminuição da ecogenicidade (seta). 


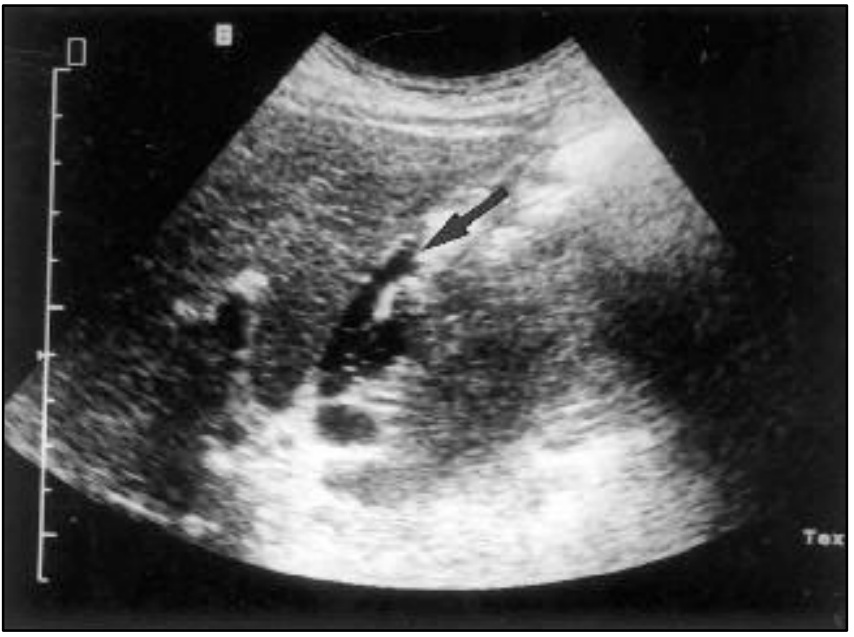

Figura 3. Ultra-sonografia em corte longitudinal ao nível do lobo hepático esquerdo. Evidencia-se líquido na pequena cavidade dos epíploons (seta).

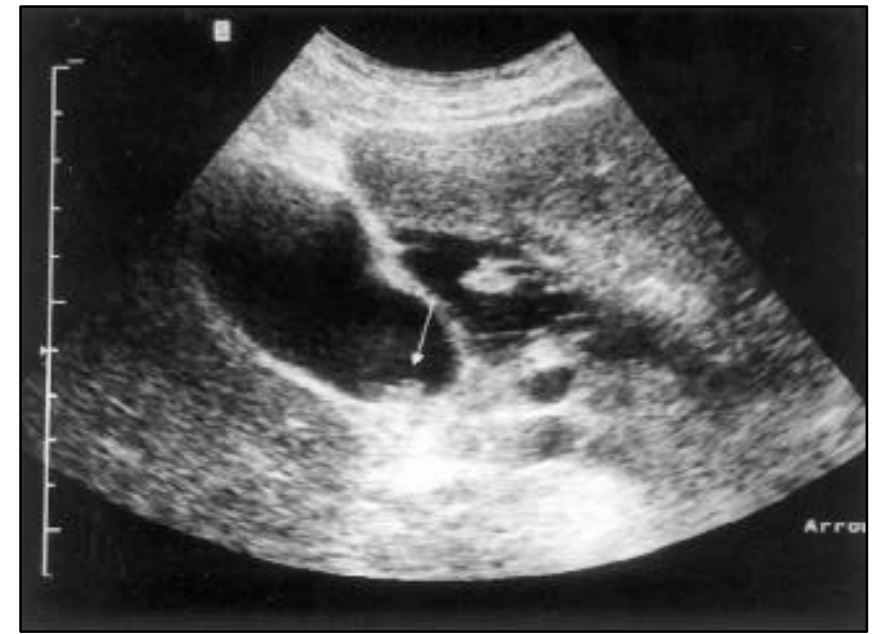

Figura 4. Ultra-sonografia abdominal ao nível do hipocôndrio direito. Observam-se microcálculos na vesícula biliar (seta), que se aglomeravam às mudanças de decúbito.
De qualquer forma, pacientes com escore menor que 3 nos critérios de Ranson e menor que 8 nos critérios de Apache II tendem a evoluir favoravelmente, apresentando formas "leves" de pancreatite aguda. Este fato pôde ser observado em nossos casos, em que todos os pacientes apresentaram resolução completa do quadro de pancreatite aguda. Mesmo os três pacientes que apresentaram pequena quantidade de líquido na pequena cavidade dos epíploons também evoluíram sem outras intercorrências.

Do ponto de vista ultra-sonográfico, o pâncreas na pancreatite aguda leve (edematosa) apresenta-se com aumento de suas dimensões e com hipoecogenicidade associada, sendo que, muitas vezes, seus contornos são mal definidos. A presença de líquido pode ser identificada na região peripancreática, especialmente na pequena cavidade dos epíploons. Ocasionalmente, a alteração inflamatória pode estender-se para outras localidades, como os tecidos adjacentes retroperitoneais, mesocólon transverso, espaço pararrenal anterior esquerdo e, mais raramente, para o mesentério e pelve, embora estas alterações sejam mais comuns em formas mais graves de pancreatite aguda $^{(\mathbf{1 0 , 1 1 )}}$. Por vezes, mesmo esses casos de pancreatite leve (edematosa) podem evoluir para formas clínicas mais graves, apresentando complicações como a presença de coleções peripancreáticas ou retroperitoneais significativas, que se apresentam com aspecto anecóico, podendo apresentar ecos em suspensão ou "débris". Deve ser notado que a ultra-sonografia constitui-se em método de grande auxílio no acompanhamento dessas formas leves de pancreatite aguda, demonstrando a evolução mais habitual desses casos, que é a resolução, tanto do quadro pancreático, como das possíveis complicações (como as coleções líquidas) ${ }^{(\mathbf{1 0}, 11)}$.

Dos pacientes estudados no presente trabalho, identificamos hipoecogenicidade do pâncreas em 19 pacientes $(90,5 \%)$, tendo sido observado, também, líquido na pequena cavidade dos epíploons em três destes pacientes $(15,8 \%)$. Dentre os pacientes nos quais foi identificada hipoecogenicidade da glândula pancreática (19 pacientes), esta hipoecogenicidade foi difusa em $73,7 \%(14 / 19)$, mais acentuada na cabeça em $10,5 \%$ (2/19), e no corpo/cauda em $15,8 \%$ (3/19). Estes dados indicam que o exame ultra-sonográfico, realizado buscando-se identificar a hipoecogenicidade na pancreatite aguda leve, pode identificar alterações na maioria dos pacientes. Entretanto, deve ser mencionado que nossa casuística não é numerosa e representa pacientes que permaneceram internados, o que certamente indica certo viés na seleção da amostra analisada. Outros autores relataram que na pancreatite aguda leve a hipoecogenicidade e o aumento glandular poderiam ser difusos em cerca de $52 \%$ dos casos e focais em cerca de $28 \%^{(\mathbf{1 0 , 1 2})}$.
Alguns autores consideram que em cerca de $20 \%$ dos casos de pancreatite aguda leve podemos não encontrar alteração da ecogenicidade pancreática ${ }^{(\mathbf{1 0}, 13)}$. No presente trabalho, em 9,5\% dos pacientes não encontramos alteração da ecogenicidade pancreática.

A maioria dos casos de pancreatite aguda é causada por cálculos biliares ou por alcoolismo. Alguns podem resultar de hipercalcemia, trauma, hiperlipidemia, origem medicamentosa, pâncreas divisum e predisposição genética. Quando não são identificados fatores que possam ser implicados na sua gênese, são considerados como de origem idiopática ${ }^{(\mathbf{1})}$.

Em dois dos nossos pacientes $(9,5 \%)$ não foi identificado qualquer fator que pudesse ser responsável pelos quadros de pancreatite aguda, tendo sido considerados como de etiologia idiopática. Outros autores referem que até $30 \%$ dos casos de pancreatite aguda podem não ter etiologia definida $^{(\mathbf{1 4})}$.

Segundo alguns autores, $40 \%$ dos casos de pancreatite aguda estão associados a cálculos biliares ${ }^{(\mathbf{1 , 5}-7)}$. O mecanismo etiológico parece estar relacionado à obstrução transitória da papila de Vater e ducto pancreático por cálculos. Embora a coledocolitíase seja encontrada em apenas $25 \%$ dos casos, em $90 \%$ dos pacientes pode-se observar a passagem de cálculos biliares para o duodeno e alças de delgado (em um período de dez dias a partir do início do 
quadro agudo de dor abdominal), pela identificação dos cálculos nas fezes ${ }^{(\mathbf{1 , 5}-7)}$.

A etiologia alcoólica seria responsável por cerca de outros $40 \%$ de casos de pancreatite aguda. Habitualmente, esses pacientes são contumazes usuários de bebidas alcoólicas destiladas, sendo que comumente apresentam história de mais de seis anos de ingestão alcoólica ${ }^{(\mathbf{1 , 5}-7)}$.

Em nossos casos pudemos demonstrar a presença de colecistopatia calculosa em 15 pacientes $(71,5 \%)$, sendo que em outros quatro pacientes $(19,0 \%)$ havia história de alcoolismo crônico e não foram identificados cálculos na vesícula biliar.

A hipercalcemia encontra-se mais comumente relacionada ao hiperparatireoidismo, embora as outras causas de hipercalcemia possam também estar associadas. Postula-se que as concentrações aumentadas de cálcio no suco pancreático possam ativar prematuramente as proteases. As hiperlipidemias primárias também podem ser causadoras desses episódios agudos, e nesses casos recomenda-se controle dietético e medicamentoso dos níveis lipídicos, para que se possa prevenir a recorrência dos ataques ${ }^{(\mathbf{1 , 5}-7)}$. Temos ainda as formas familiares de pancreatite aguda, usualmente com início na infância. A presença de diabetes e esteatorréia é comum nesses pacientes. $\mathrm{O}$ adenocarcinoma pancreático apresenta ocorrência aumentada nos pacientes com pancreatite familiar ${ }^{(\mathbf{1})}$. Ainda com relação à etiologia, algumas drogas podem estar relacionadas com o desenvolvimento de pancreatite aguda, como os corticosteróides, anticoncepcionais orais, azatioprina e diuréticos tiazídicos ${ }^{(\mathbf{1 , 5}-7)}$.

Ainda devemos mencionar o pâncreas divisum, que pode predispor à ocorrência de pancreatite aguda obstrutiva. Nos casos em que esta anormalidade esteja presente, e possivelmente associada a estreitamento da abertura da papila duodenal menor, o orifício poderia ser pequeno para dar passagem ao fluido pancreático. $\mathrm{O}$ seu diagnóstico é realizado pela colangiopancreatografia endoscópica retrógrada, e mais recentemente poderia ser obtido pela co- langiografia por ressonância magnética. Nos pacientes em que essa anormalidade é encontrada, e nenhum outro fator etiológico estiver relacionado, é razoável considerar a anormalidade de drenagem pancreática como causa dos episódios de pancreatite aguda $^{(\mathbf{1 , 5}-7)}$.

Em um pequeno número de pacientes o diagnóstico de pancreatite aguda permanece puramente clínico, uma vez que os exames de imagem não mostram nenhuma alteração. Muitas publicações avaliam que $14 \%$ a $28 \%$ dos exames de imagem podem ser normais nos casos de pancreatite aguda ${ }^{(10,11,15-17)}$. Estes valores ainda estão sujeitos a maior variação, decorrente das variadas formas de apresentação que a pancreatite aguda pode assumir, e ainda pela falta de correlação anatomopatológica em um número significativo dos casos publicados na literatura. Conforme referido anteriormente, e de conformidade com estes estudos anteriores, em nossos casos não encontramos alterações ultra-sonográficas em dois pacientes $(9,5 \%)$.

A presença de líquido (identificado em três dos nossos pacientes com hipoecogenicidade pancreática associada-15,8\%) na pequena cavidade dos epíploons reitera a importância da ultra-sonografia. Dessa forma, mesmo em casos mais leves, poderemos ter a presença de coleções líquidas, que poderiam ter passado despercebidas por um exame mais desatento. Estas coleções devem ser acompanhadas, na medida em que poderiam ser solo fértil para o aparecimento de complicações, como as infecções. Em nossos casos, as coleções líquidas desapareceram em um período máximo de 14 dias a partir do início do quadro, e os pacientes evoluíram sem qualquer outra complicação.

\section{CONCLUSÃO}

Os autores concluem que a ultra-sonografia representa um importante método na avaliação dos pacientes com pancreatite aguda leve, podendo identificar alterações ultra-sonográficas na maioria deles, e, ain- da, identificar e acompanhar a evolução de coleções líquidas peripancreáticas.

\section{REFERÊNCIAS}

1. Reber HA, Way LW. Pancreas. In: Way LW, ed. Current surgical diagnosis \& treatment. Connecticut: Appleton \& Lange, 1994:567-94.

2. Ranson JHC. Etiological and prognostic factors in human acute pancreatitis: a review. Am J Gastroenterol 1982;77:633-8.

3. Knaus WA, Draper EA, Wagner DP, Zimmerman JE. APACHE II: a severity of disease classification system. Crit Care Med 1985;13:818-29.

4. Dervenis C, Johnson CD, Bassi C, et al. Diagnosis, objective assessment of severity, and management of acute pancreatitis. Santorini consensus conference. Int J Pancreatol 1999;25:195210.

5. Carey LC. The etiology of pancreatitis. In: Moody FG, ed. Surgical treatment of digestive disease. Chicago: Year Book Med Publ, 1990:459-69.

6. Ranson JHC. The diagnosis and treatment of acute pancreatitis. In: Moody FG, ed. Surgical treatment of digestive disease. Chicago: Year Book Med Publ, 1990:471-86.

7. Friedman LS. Liver, biliary tract \& pancreas. In: Tierney LM Jr, McPhee SJ, Papadakis MA, eds. Current medical diagnosis \& treatment. Connecticut: Appleton \& Lange, 1998:628-65.

8. Ranson JHC, Spencer FC. The role of peritoneal lavage in severe acute pancreatitis. Ann Surg 1978;187:565-75.

9. Ranson JHC, Rifkind KM, Turner JW. Prognostic signs and nonoperative peritoneal lavage in acute pancreatitis. Surg Gynecol Obstet 1976;143: 209-19.

10. Ferreyra NP, Athaide ACM, Vezozzo DCP, Rocha DC, Cerri GG. Pâncreas. In: Cerri GG, Rocha DC, eds. Ultra-sonografia abdominal. São Paulo: Sarvier, 1993:143-68.

11. Weill FS. Ultrasound diagnosis of digestive diseases. 4th ed. Berlin: Springer-Verlag, 1996:43168.

12. Doust BD, Pearce JD. Gray-scale ultrasonic properties of the normal and inflamed pancreas. Radiology 1976;120:653-7.

13. Hill MC. Pancreatic sonography: an update. In: Saunders RC, ed. Ultrasound annual, 1982. New York: Raven Press, 1982.

14. Chebli JMF, Martins Júnior EV, Gaburri AK, Ferreira LE, Gil JZ, Neves MM. Microcristais e "barro biliar": patogênese e significado clínico. Arq Gastroenterol 1996;33:232-43.

15. Balthazar EJ, Ranson JHC, Naidich DP, Megibow AJ, Caccavale R, Cooper MM. Acute pancreatitis: prognostic value of CT. Radiology 1985;156: 767-72.

16. Jeffrey R, Freeny PC, Mueller P. Percutaneous drainage of infected pancreatic fluid collections. Perspect Radiol 1988;1:69-76.

17. Ammann R, Munch R, Largiader F, Akovbiantz A, Marincek B. Pancreatic and hepatic abscesses: a late complication in 10 patients with chronic pancreatitis. Gastroenterology 1992;103:560-5. 